\title{
Identification of recurrence-related genes by integrating microRNA and gene expression profiling of gastric cancer
}

\author{
ZHI YAN ${ }^{1}$, YIMIN XIONG $^{1}$, WEITIAN XU ${ }^{1}$, MIN LI $^{1}$, YI CHENG $^{1}$, \\ FANG CHEN $^{2}$, SHIFANG DING ${ }^{3}$, HUALIN XU ${ }^{1}$ and GUORONG ZHENG ${ }^{1}$ \\ Departments of ${ }^{1}$ Digestive Diseases, ${ }^{2}$ Medical Laboratory, and ${ }^{3}$ Cardiology, \\ Wuhan General Hospital of Guangzhou Command, Wuchang, Wuhan 430070, P.R. China
}

Received July 2, 2012; Accepted August 17, 2012

DOI: $10.3892 /$ ijo.2012.1637

\begin{abstract}
We previously analyzed the microRNA (miRNA) expression pattern in gastric cancer with and without recurrence and obtained 17 differentially expressed miRNAs with potential to predict recurrence risk for GC patients. In the present study, we aimed to investigate recurrence-related genes which may be regulated by the differentially expressed miRNAs identified in our prior research. Three different miRNA target gene databases (miRanda, TargetScan and PicTar) were used for searching the potential genes regulated by miRNAs. A combination was performed between miRNA target genes and recurrence-related gene expression profiling. Three bioinformatics algorithms (PAM, SVM and RF) were used to feature recurrence-related gene selection. In addition, we validated the expression levels of the genes in GC patients using real-time PCR. A total of 3,263 genes were identified as potential targets of 17 miRNAs. We identified 2,736 differential expressed genes using the SAM method based on $22 \mathrm{~K}$ oligo microarray data which included 7 recurrence and 4 without recurrence GC samples. Combining the target genes regulated by miRNAs and the differentially expressed genes between recurrence and non-recurrence groups, we identified 228 differential genes for further study. Finally, we identified HNRPA0 and PRDM4 as risk biomarkers of GC patients, which were regulated by hsa-miR-194 and hsa-miR-373, respectively. Our data indicated that HNRPA0 and PRDM4 may be involved in the recurrence process of GC and have potential to act as new prognostic biomarkers in predicting recurrence risk for gastric cancer patients.
\end{abstract}

Correspondence to: Dr Guorong Zheng, Department of Digestive Diseases, Wuhan General Hospital of Guangzhou Command, Wuluo Road 627, Wuchang, Wuhan 430070, P.R. China

E-mail: guorongzheng@sina.com

Key words: gastric cancer, recurrence, gene expression profiling, microRNAs, prognostic biomarkers

\section{Introduction}

Gastric cancer (GC) is one of the most frequent tumors and the second leading cause of mortality worldwide (1). Since 1976, gastric cancer has increased 4-10\% per year among men in the United States, more rapidly than any other type of cancer. Surgery is still the main option for treating GC, however, even after performing curative resection approximately 40-65\% of GC patients will experience a recurrence of the disease possibly encompassing local relapse, peritoneal dissemination or hematogenous metastasis $(2,3)$. The high recurrence rate of $\mathrm{GC}$ is the main reason to treatment failure and once it happens, the quality of life and survival will decrease greatly. Therefore, identification of biomarkers with potential for understanding molecular mechanisms and recognizing the biological characteristics involved in recurrence is a key for early evaluation of the prognosis in GC patients.

In 2011, we reported that the combination of a group of microRNAs (miRNAs) as a predictor has potential to predict recurrence risk in gastric cancer patients following surgical resection (4). In our previous study, we used miRNA microarray and bioinformatics methods to characterize recurrence-related biomarkers from primary tumor samples, and identified 17 differential miRNAs including 10 upregulated and 7 downregulated miRNAs in recurrence group. This miRNA profiling provided us a with a powerful resource for better understanding the molecular mechanism within GC recurrence.

The regulation by miRNAs is one of the important modes for alterations of gene or protein. Compared with miRNAs, more advantageous in clinical diagnosis and adjuvant therapy were proved by using genes or proteins as signature. Several factors including gene expression changes related with recurrence have been reported $(5,6)$. In addition, a few predictive or diagnostic methods have been used to evaluate the recurrence risk based on gene expression profiling or a set of clinical variables (7-10). This led us to believe that combination miRNAs expression profile, targeted genes and gene expression profile may obtain more accurate genes to predict recurrence. Thus, we set out to investigate recurrence-related genes by intergration of miRNAs and gene expression profiling in advanced gastric cancer. 
In this study, we predicted the targeted genes which plausibly are regulated by the identified miRNAs using miRanda, TargetScan and PicTar and obtained 3,263 predicted miRNA targets. At the same time, a recurrence related gene expression profile based on 11 GC microarray data was constructed for combination with miRNA targeted genes. A total of 228 different genes were identified with the criteria of $\mathrm{P}<0.05$, fold-change $>2.0$. We further integrated three bioinformatics methods to extract genes with characteristic expression and finally obtain a single gene set (HNRPA0 and PRDM4) which have potential to predict recurrence risk in GC.

\section{Materials and methods}

Clinical samples. Patients undergoing gastrectomy for potentially curable GC at the Wuhan General Hospital of Guangzhou Command were subjects in this study. Eligibility for inclusion in this study included histologically confirmed adenocarcinoma of the stomach or gastroesophageal junction. All patients had received complete resections including an attempt at complete tumor removal with inclusion of wide negative margins and extended retroperitoneal lymphadenectomy (D2 type). Information on clinicopathological, therapeutic and outcome parameters of patients from May 2005 to June 2010 was collected retrospectively. Cancer staging was performed according to the fifth edition of the American Joint Commission on Cancer TNM criteria.

Recurrence was defined as any cancer recurrence including lymph node, remnant stomach, local, peritoneal and hematogenous metastases over 1 year after surgical operation. All patients that experienced recurrence of cancer were diagnosed clinically or radiographically and confirmed by biopsy via upper gastrointestinal endoscope or percutaneous puncture. The radiographic standard for the recurrence diagnosis included CT or MRI of the chest, abdomen, pelvis, head and bone scans, or other diagnostic tests which were used only under special circumstances. All of the samples were obtained from surgical specimens of patients with gastric adenocarcinoma and all patients gave written consent for the use of these tissues for research purposes. We selected samples from 48 patients with and without GC recurrence.

This study has been approved by the Ethics Committee of the Wuhan General Hospital of Guangzhou Command, PLA. All gastric cancer patients provided written informed consent in our study.

Prediction of miRNA targeted genes. We utilized three different databases to select plausible targets of the differential expressed miRNAs: miRanda (http://www.microrna.org), TargetScan (http://www.targetscan.org) and PicTar (http:// pictar.mdc-berlin.de). To identify which genes were most likely targeted with the given miRNAs, we integrated the results come from the different databases.

Microarray experiments. The microarray experiments were performed as described in detail on the website of CapitalBio (http://www.capitalbio.com). A Human Genome Oligo Set Version 2.1 consisting of about 22,000 human genes was purchased from Qiagen Operon Co. A total of $11 \mathrm{GC}$ samples were selected for microarray experiment, including
7 and 4 samples with and without recurrence, respectively. Total RNA was extracted with TRIzol reagent (Invitrogen, Gaithersburg, MD) and further purified with a NucleoSpin RNA Clean-up kit (Macherey-Nagel, Germany). Fluorescent dye (cy5 and cy3-dCTP) labeled DNA was produced through an RNA amplification method and subsequently followed the method previously published (11). Arrays were scanned with a confocal Lux Scanner and images were analyzed with two-channal microarray technology, fluorescent dye-labeled cDNA from each GC samples were pooled to hybridize with one chip and hybridization was performed in duplicate with dye-reversal approach. Only spots with intensity in at least one channel exceeding the local background signal plus 3 standard deviations were accepted for further analysis. Then a space and intensity-dependent normalization based on a LOWESS in the R language package (http://www.R-project. org) was employed to normalize the two-channel ratio value.

Unsupervised algorithms. Significance analysis of microarrays (SAM) was used to perform the unsupervised calculation. SAM is a statistical technique based on t-test for finding significant genes in a set of microarray experiments. It was proposed by Tusher et al (12). SAM computes a statistic $d_{i}$ for each gene $i$, measuring the strength of the relationship between gene expression and the response variable. It uses repeated permutations of the data to determine if the expression level of some genes were significantly related to the response. The cut-off for significance is determined by a tuning parameter delta, chosen by the user based on the false positive rate. A fold-change parameter can also be chosen to ensure that the called genes change at least a pre-specified amount. Hierarchical clustering of the differential expressed genes was performed with Cluster 3.0 version and Genesis using the average linkage algorithm.

Supervised machine learning algorithms. For the purpose of selecting feature genes, as well as classifying observations precisely, we applied various kinds of machine learning algorithms. Prediction analysis of microarrays (PAM) is a statistical technique for class prediction from gene expression data using nearest shrunken centroids. The method of nearest shrunken centroids identifies subsets of genes that best characterize each class (13).

Support vector machine (SVM) is one of the most classic supervised learning algorithms, useful for recognizing subtle patterns in complex datasets (14). It has been successfully applied to the classification of cancer tissue samples based on microarray expression data (15). The algorithm performs discriminative classification, learning by example to predict the classifications of previously unclassified data. In principle, the SVM can be applied to very high dimensional data without altering its formulation. Such capacity is well suited to the microarray data structure. In our study, we used Bhattacharyya distance as classification index and SVM as classifier to perform the feature selection. Leave-one-out cross validation (LOOCV) was used to validate the classification accuracy.

Random forests (RF) is one of the most important supervised methods for feature gene selection (16-18). During the classifying process, RF returns several measures of vari- 
Table I. Details of patients and tumors used in this study.

\begin{tabular}{|c|c|c|c|}
\hline Characteristics & Recurrence group $(n=23)$ & Non-recurrence group ( $\mathrm{n}=25$ ) & P-value \\
\hline \multicolumn{4}{|l|}{ Gender } \\
\hline Male & 18 & 15 & \\
\hline Female & 5 & 10 & 0.173 \\
\hline \multicolumn{4}{|l|}{ Age (year) } \\
\hline Median & 64 & 56 & 0.231 \\
\hline Range & $30-83$ & $33-79$ & \\
\hline \multicolumn{4}{|l|}{ Tumor location } \\
\hline Cardia & 8 & 4 & 0.318 \\
\hline Body & 4 & 5 & \\
\hline Pyloric antrum & 11 & 16 & \\
\hline \multicolumn{4}{|l|}{ Differentiation } \\
\hline Poor & 13 & 15 & 0.971 \\
\hline Moderate-poor & 6 & 6 & \\
\hline Others & 4 & 4 & \\
\hline \multicolumn{4}{|c|}{ Lymph node resection } \\
\hline$<12$ & 20 & 25 & 0.062 \\
\hline$>12$ & 3 & 0 & \\
\hline \multicolumn{4}{|l|}{ UICC stage } \\
\hline I & 2 & 5 & 0.108 \\
\hline II & 2 & 5 & \\
\hline III & 16 & 15 & \\
\hline $\mathrm{IV}(\mathrm{M} 0)$ & 3 & 0 & \\
\hline \multicolumn{4}{|l|}{ Embolus } \\
\hline With & 9 & 3 & 0.03 \\
\hline Without & 14 & 22 & \\
\hline \multicolumn{4}{|c|}{ Adjuvant chemotherapy } \\
\hline Performed & 10 & 12 & 0.967 \\
\hline Not performed & 13 & 13 & \\
\hline \multicolumn{4}{|l|}{ Patients' status } \\
\hline Survival & 7 & 20 & $<0.001$ \\
\hline Death & 16 & 5 & \\
\hline $\begin{array}{l}\text { Median survival } \\
\text { time (month) }\end{array}$ & 23.4 & 61.1 & $<0.001$ \\
\hline
\end{tabular}

able importance. The most reliable measure is based on the decrease of classification accuracy when values of a variable in a node of a tree are permuted randomly.

Receiver operating characteristic (ROC) curves and statistical analyses. ROC curves (MedCalc, 8.2.1.0 version, Mariakerke, Belgium) were used to analyze the classification sensitivity and specificity of the feature genes based on test samples. The $\mathrm{Ct}$ values of each sample after real-time PCR were used to perform the ROC analysis. The clinical data were analyzed using the t-test, with $\mathrm{P}<0.05$ considered statistically significant. The survival curve study was also analyzed by MedCalc.
RNA isolation and real-time PCR. We collected 37 human GC tissues from the Wuhan General Hospital of the Guangzhou Military Command for real-time PCR experiment, including 16 GC samples with and 21 without recurrence. Total RNA was extracted from the tissue samples according to standard TRIzol protocol (Invitrogen, Carlsbad, CA, USA). Total RNA $(5 \mu \mathrm{g})$ was reverse transcribed to cDNA with $200 \mathrm{U}$ M-MLV reverse transcriptase (Promega, Madison, WI, USA) according to a standard manufacturer's protocol. RT reaction conditions were used: $37^{\circ} \mathrm{C}$ for $60 \mathrm{~min}, 72^{\circ} \mathrm{C}$ for $10 \mathrm{~min}$. Q-RT-PCR was performed in a total $20 \mu \mathrm{l}$ reaction mixture $2 \mu \mathrm{l}$ of cDNA, $0.6 \mu 1$ 20X EvaGreen (CapitalBio Corp., Beijing, China), $0.5 \mu 1$ of each $10 \mu \mathrm{M}$ forward and reverse primers, $0.5 \mu 1$ of $2.5 \mathrm{mM}$ dNTP, 1.5 U Cap Taq polymerase (CapitalBio Corp.), $10 \mu 12 \mathrm{X}$ 
Table II. Differential expressed genes regulated by identified microRNAs.

\begin{tabular}{llc}
\hline Expressed level & \multicolumn{1}{c}{ microRNAs } & $\begin{array}{c}\text { No. of target } \\
\text { genes }\end{array}$ \\
\hline Up in recurrence & hsa-miR-128b & 433 \\
samples & hsa-miR-133a/b & 302 \\
& hsa-miR-375 & 161 \\
& hsa-miR-200b/c/429 & 484 \\
& hsa-miR-194 & 207 \\
& hsa-miR-335 & 146 \\
& hsa-miR-422b & 477 \\
Down in recurrence & hsa-miR-19a & 527 \\
samples & hsa-miR-373 & 298 \\
& hsa-miR-217 & 159 \\
& hsa-miR-142-3p & 162 \\
& hsa-miR-142-5p & 307 \\
& hsa-miR-32 & 567 \\
Total & hsa-miR-296 & 122 \\
& & 17
\end{tabular}

PCR buffer for EvaGreen and $6.1 \mu \mathrm{l}$ of $\mathrm{H}_{2} \mathrm{O}$. Quantification of differentially expressed genes was conducted with an RT-Cycler ${ }^{\mathrm{TM}} 2.0$ system (CapitalBio Corp.). Q-RT-PCR was carried out with programmed parameters, heating at $95^{\circ} \mathrm{C}$ for $5 \mathrm{~min}$ followed by 40 cycles of a three-stage temperature profile of $95^{\circ} \mathrm{C}$ for $30 \mathrm{sec}, 57^{\circ} \mathrm{C}$ for $30 \mathrm{sec}, 72^{\circ} \mathrm{C}$ for $30 \mathrm{sec}$. The expression of each gene/miRNAs was normalized with $\beta$-actin/U6 snRNA expression and according to the $2^{-\Delta \Delta \mathrm{Ct}}$ formula (19).

\section{Results}

Clinical characteristics of GC patients. A total of 48 patients with/without recurrence were selected for systematic analysis. Twenty-three patients had recurring GC that was proven pathologically by biopsy at anastomosis sites via endoscopy. Twenty-five patients without recurrence were selected as the control group with matches in gender, age at diagnosis, TNM staging, treatment and the number of involved lymph node (Table I).

There was no significant difference in the gender $(\mathrm{P}=0.173)$, age $(\mathrm{P}=0.231)$, tumor location $(\mathrm{P}=0.318)$, differentiation $(\mathrm{P}=0.971)$, lymph node resection $(\mathrm{P}=0.062)$, UICC stage $(\mathrm{P}=0.108)$ and status of adjuvant chemotherapy $(\mathrm{P}=0.967)$. There was a significant difference in survival/death ratio noted (7/16 in recurrence group vs. 20/5 in non-recurrence group, $\mathrm{P}<0.001$ ), with median survival time of 23.4 months in recurrence vs. 61.1 months in non-recurrence group respectively $(\mathrm{P}<0.001)$ (Table I).

Identification of genes plausibly regulated by recurrencerelated miRNAs. We previous analyzed the miRNAs expression in 4 recurrence and 4 non-recurrence GC patients. A total of 17 miRNAs were identified as candidate biomarkers related to the recurrence risk of GC. We searched for putative 17 miRNAs targeted genes employing the most widely used programs including miRanda, TargetScan and PicTar, and obtained 4,352 plausible targeted genes, including 1,089 genes regulated by more than two miRNAs. Finally, a total of 3,263 targeted genes were focused for next study (Table II).

Meanwhile, a total of 2,736 genes were differential expressed in recurrence compared with non-recurrence group with a criteria of $\mathrm{P}<0.05, \mathrm{FC}>1.0$. Combined with the differential expressed genes and the miRNAs targeted genes,

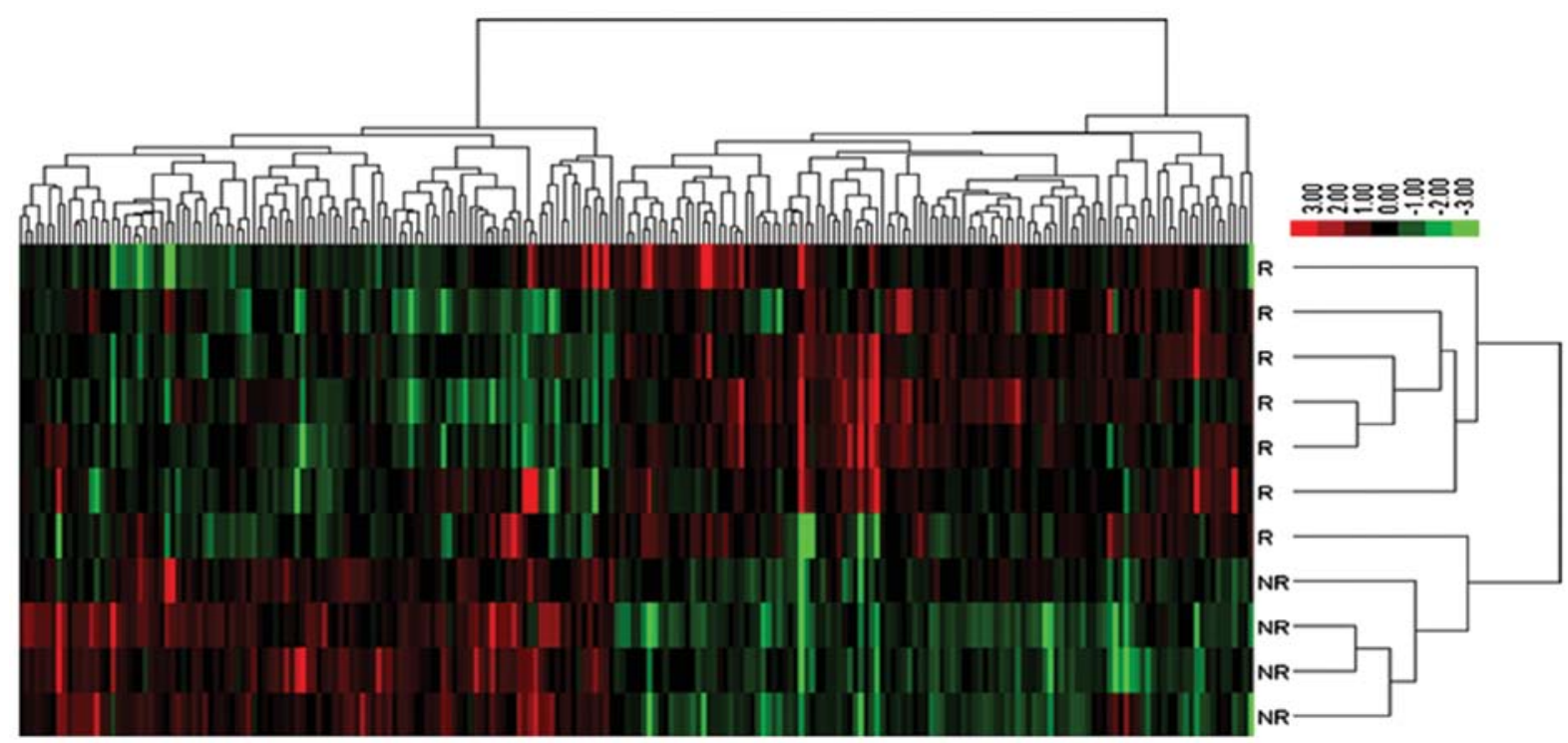

Figure 1. Graphical overview of differentially expressed genes in gastric with/without recurrence. A total 228 differential expressed genes were identified by BAGEL in gastric cancer with/without recurrence, according to the criteria of $q<0.05$. Hierarchical clustering of the data matrix consists of 228 differential expressed genes by 7 gastric cancers with recurrence and 4 without recurrence, including 115 genes upregulated and 113 downregulated in recurrence samples. Columns represent samples and rows represent genes (black, green and red correspond to no-change, downregulated and upregulated, respectively). NR, non-recurrence; R, recurrence. 

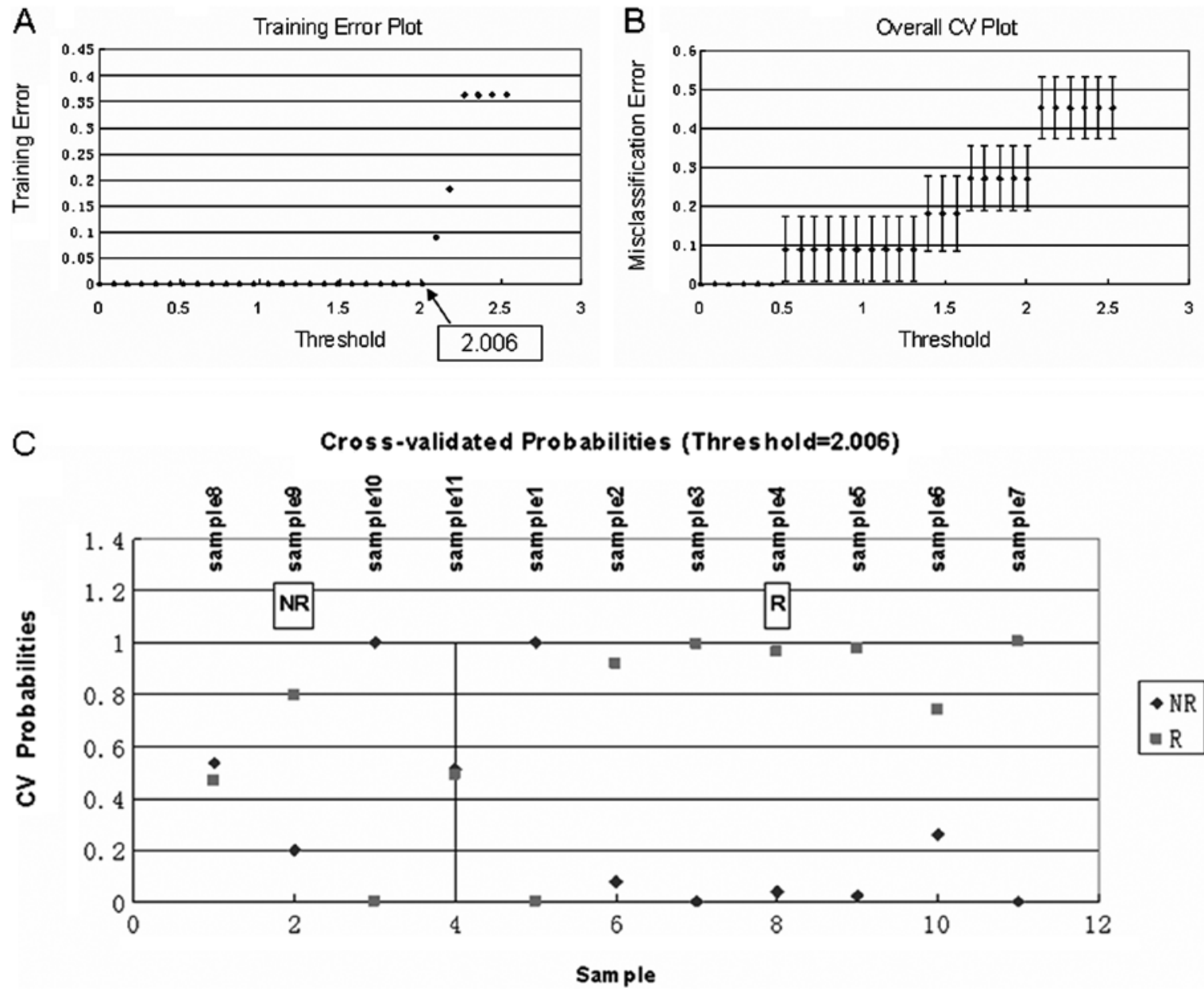

Figure 2. Feature genes selection based on PAM. (A) The lowest training error was equal to 0 when threshold value equal to 2.006 , according to a classifier consisted of 11 genes. (B) The overall cross validation results showed that the lowest training error was equal to 0.273 when threshold value equal to 2.006 . (C) Three samples were misclassified using the 11-gene-classifier in the process of cross validation.

Table III. Feature genes selection based on PAM.

\begin{tabular}{llcc}
\hline Genes & \multicolumn{1}{c}{ ACC } & R score & NR score \\
\hline SH2D3C & NM_005489 & 0.1668 & -0.2918 \\
ANKRD12 & NM_015208 & 0.1474 & -0.258 \\
ZCWPW1 & NM_017984 & -0.1168 & 0.2044 \\
BMP1 & NM_006129 & -0.1102 & 0.1929 \\
CABLES2 & BC003122 & 0.1085 & -0.1899 \\
GLCCI1 & AK055741 & 0.0812 & -0.1421 \\
AK2 & AK023758 & -0.0665 & 0.1163 \\
HNRPA0 & U23803 & -0.0621 & 0.1086 \\
ESRRG & NM_001438 & 0.0316 & -0.0554 \\
PRO0149 & NM_014117 & -0.0269 & 0.0471 \\
PRDM4 & NM_012406 & 0.0048 & -0.0084 \\
\hline
\end{tabular}

we obtained 228 genes which were probably regulated by miRNAs. Hierarchical clustering of the data matrix consist of 228 genes is shown in Fig. 1.

Identification of a two-gene signature to distinguish recurrence from non-recurrence GC samples. For these 228 differentially
Table IV. Feature genes selection based on SVM.

\begin{tabular}{lcc}
\hline Value of BD & No. of genes & Correct rate $(\%)$ \\
\hline $\mathrm{t}=0.8$ & 1 & 100 \\
$\mathrm{t}=0.7$ & 8 & 100 \\
$\mathrm{t}=0.6$ & 16 & 63.64 \\
$\mathrm{t}=0.5$ & 27 & 63.64 \\
$\mathrm{t}=0.4$ & 43 & 63.64 \\
$\mathrm{t}=0.3$ & 90 & 63.64 \\
$\mathrm{t}=0.2$ & 184 & 63.64 \\
$\mathrm{t}=0.1$ & 223 & 63.64 \\
$\mathrm{t}=0$ & 228 & 63.64 \\
\hline
\end{tabular}

expressed genes, we used PAM, SVM and RF approaches for supervised classification and selecting feature genes. A total of 11,9 and 10 genes were selected as best classifiers using PAM (Fig. 2 and Table III), SVM (Fig. 3A and B and Table IV) and RF (Fig. 3C and Table V), respectively. We integrated the results from the 3 approaches, and identified a two-gene signature (HNRPA0 and PRDM4) which has potential to classify the recurrence and non-recurrence gastric cancer samples 
A

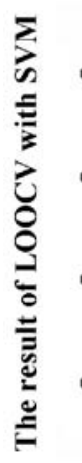

B

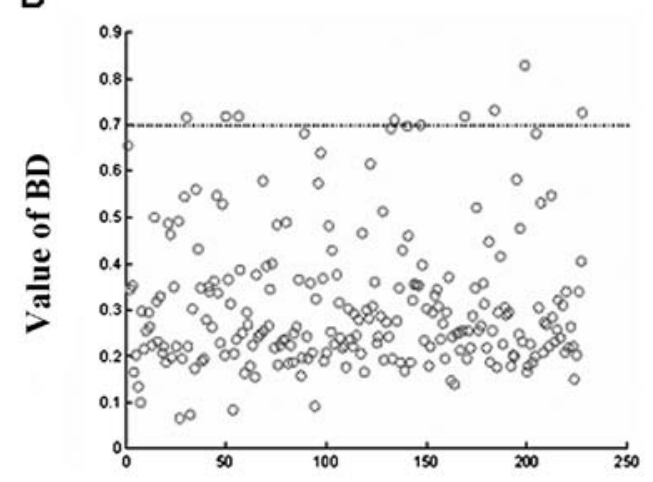

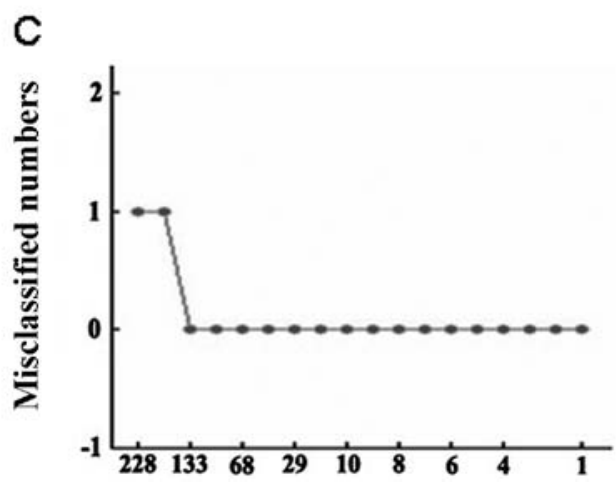

Figure 3. Graphical overview of characteristic genes selected by SVM and RF. (A and B) Nine genes were identified by SVM in gastric cancer with/without recurrence, according to the criteria of $\mathrm{q}<0.05$. (C) The lowest classification rate was equal to 0 when the classifier consist of 133 genes according to the criteria of $\mathrm{q}<0.05$. We chose 10 candidate genes with top score for further study.

Table V. Feature genes selection based on RF.

\begin{tabular}{lcccc}
\hline Genes & R score & NR score & Accuracy & Gini index \\
\hline BCR & 17.330 & 20.893 & 12.513 & 0.481 \\
HBXAP & 16.939 & 21.181 & 12.418 & 0.492 \\
NLGN1 & 16.756 & 21.109 & 12.369 & 0.463 \\
ZCWPW1 & 16.718 & 21.328 & 12.336 & 0.460 \\
CCPG1 & 16.488 & 20.349 & 12.030 & 0.462 \\
TMOD3 & 15.923 & 19.992 & 11.713 & 0.457 \\
USP6 & 16.117 & 19.605 & 11.677 & 0.444 \\
PRDM4 & 15.614 & 20.020 & 11.653 & 0.466 \\
HNRPA0 & 15.503 & 19.711 & 11.503 & 0.464 \\
ZNF533 & 15.215 & 18.938 & 11.125 & 0.477 \\
\hline
\end{tabular}

correctly (Fig. 4A and B) with a high sensitivity and specificity in microarray samples, respectively (Fig. 4C and D). Our results showed that HNRPA0 and PRDM4 were plausibly regulated by hsa-miR-194 and hsa-miR-373, respectively. The results matched our previous microarray study, hsa-miR-194 was upregulated while HNRPA0 downregulated in recurrence group; hsa-miR-373 was downregulated in recurrence group while a low expression of PRDM4 were observed in recurrence group.

Validation of the expression levels of the identified miRNAs and its target genes using quantitative real-time PCR. The relative expression levels of hsa-miR-194, hsa-miR-373,
HNRPA0 and PRDM4 were detected by real-time PCR in all the other 37 test samples (11 samples as training used for microarray) compared with the matched adjacent tissue as control. The results showed that the relative expression level of hsa-miR-194 was 10.36 in recurrence group compared to 7.83 in non-recurrence group; of HNRPA0 was 9.49 and 14.34 in recurrence compared to non-recurrence group. Similar results were observed on hsa-miR-373 and PRDM4. The relative expression level of hsa-miR-373 was 7.26 and 16.92, and PRDM4 was 25.42 and 3.11 in recurrence compared to nonrecurrence group, respectively (Fig. 5).

Combination of HNRPAO and PRDM4 as a signature to predict recurrence risk in GC patients. The $48 \mathrm{GC}$ patients were divided into two groups, including 23 patients with recurrence as a group and 25 patients without recurrence as a group. The patients who experienced a recurrence of GC had a significantly reduced median survival rate $(\mathrm{P}<0.001$; Fig. 6A). Combined with the expression levels of HNRPA0 and PRDM4 detected by real-time PCR in 37 GC test samples and the microarray data in 11 training samples, we divided our patients into two groups: HNRPA0(+)/PRDM4(-) and HNRPA0(-)/PRDM4(+), represent high expressed HNRPA0 and low expressed PRDM4 group, low expressed HNRPA0 and high expressed PRDM4 group, respectively. A significant difference of survival time was observed between HNRPA0(+)/ PRDM4(-) and HNRPA0(-)/PRDM4(+) group ( $<<0.001$; Fig. 6B). GC patients with HNRPA0(+)/PRDM4(-) had significantly reduced median and overall survival compared to those with HNRPA0(-)/PRDM4(+). 


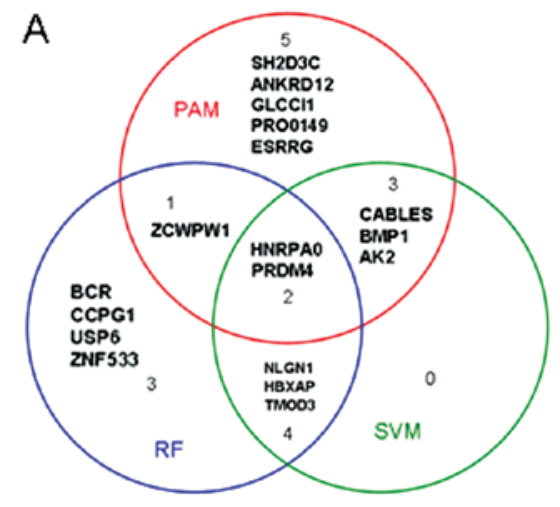

C

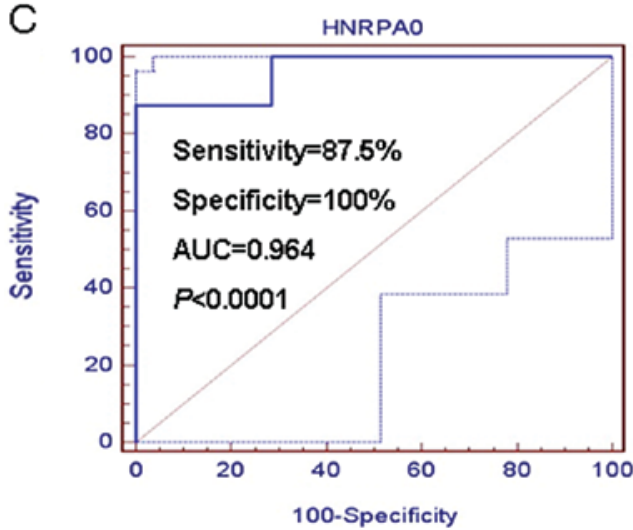

B
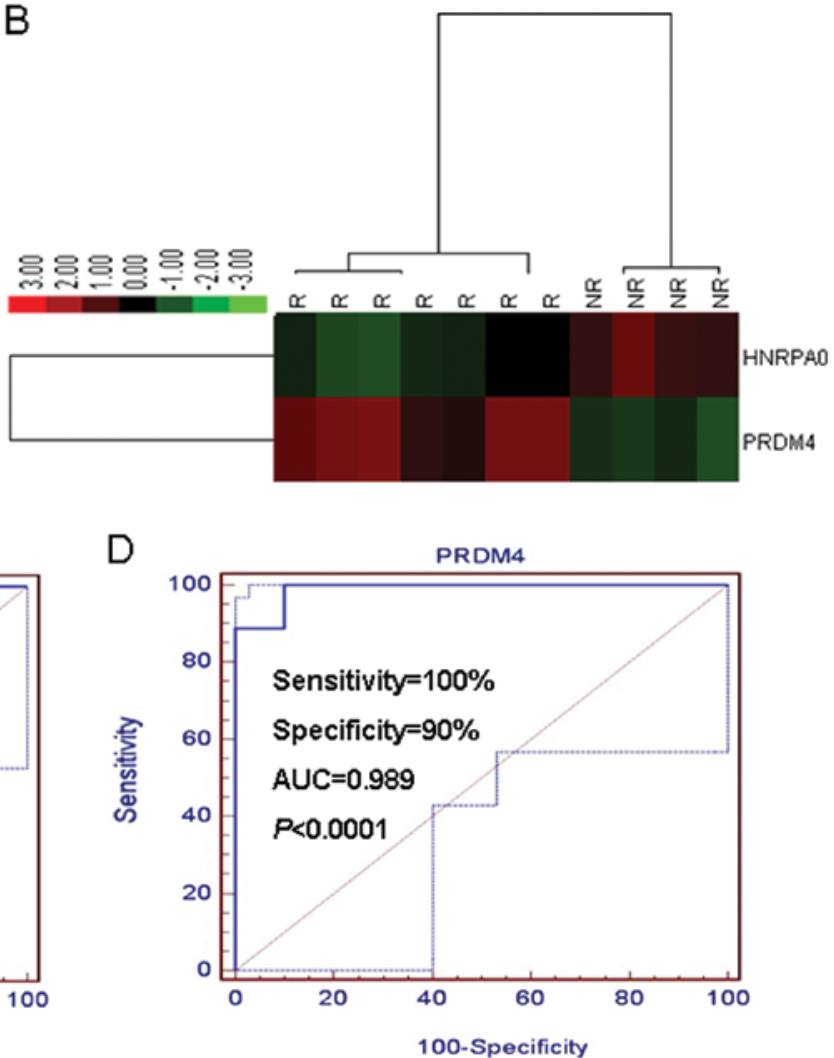

Figure 4. Identification of a two-gene signature to distinguish recurrence and non-recurrence in GC samples. (A) Combined with the three bioinformatics approaches, 2 genes (HNRPA0 and PRDM4) were identified as a specified expression pattern to classify gastric cancer with/without recurrence. (B) Graphical overview of two characteristic genes. (C and D) ROC results showed that HNRPA0 and PRDM4 were sensitive and specific in classifying recurrence and non-recurrence in GC samples.

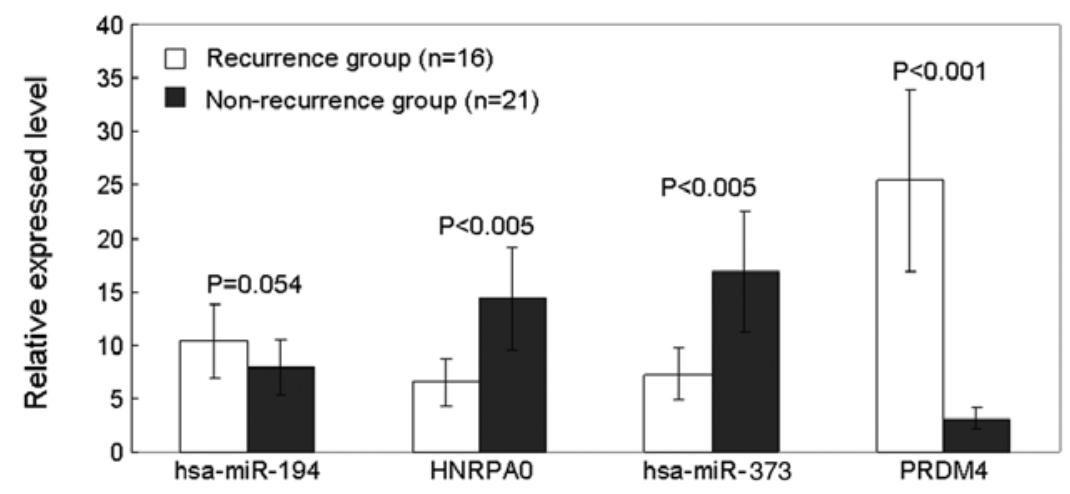

Figure 5. Analysis of differential expressed genes by real-time PCR. Gene and miRNA specific primers were designed to the hsa-miR-194, hsa-miR-373 HNRPA0 and PRDM4. $\beta$-actin and U6 snRNA were used as control to calculate the relative expression of differentially expressed miRNAs and genes by the $2^{-\triangle \Delta C T}$ method. The results showed that hsa-miR-194 was downregulated and its target gene HNRPA0 was upregulated in recurrence samples; hsa-miR-373 was upregulated and its target gene PRDM4 was downregulated in recurrence samples.

\section{Discussion}

Recurrence in early gastric cancer patients is rare, while most patients who have undergone non-curative surgery recur rapidly. Therefore, it is important to identify high-risk cases for recurrence among advanced gastric cancer patients after curative resection. Traditional clinicopathological factors are sometimes inadequate for prediction of recurrence in individuals and many research groups have attempted to identify new technologies that may distinguish high-risk cases. Many investigations documented that miRNAs alterations are involved in the initiation and progression of human cancers (20-25). Microarray technology has developed significantly and become a comprehensive and useful method to help us better understands cancers (26). miRNA expression profiling of human tumors from microarray technology has identified signatures associated with diagnosis, staging, progression, prognosis and response to treatment (27-33). Therefore, we identified a recurrence related miRNA profiling for recurrence risk prediction based on a group of Chinese GC patients.

To commence understanding how the expression changes of the 17 miRNAs affect outcome, we identified recurrence- 

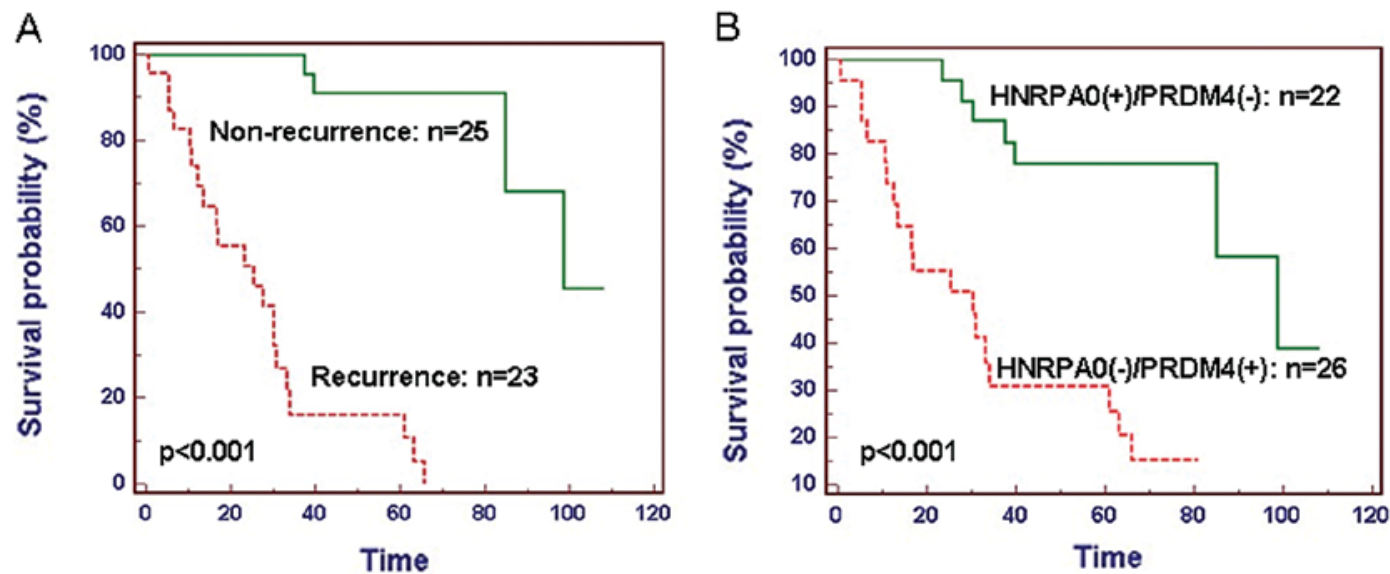

Figure 6. Survival analyses according to the expression levels of identified genes. (A) A significant difference of survival time has observed between recurrence and non-recurrence group. (B) GC patients with HNRPA0(+)/PRDM4(-) had significantly reduced median and overall survival compared to those with HNRPA0(-)/PRDM4(+).

associated miRNA-targeted genes that are differentially expressed in patients who develop recurrence. Then a combination was performed between miRNA targeted genes and gene expression profile data. In this study, we analyzed primary gastric cancer cases to predict recurrence and defined recurrence-free cases as those free of recurrence for at least one year after curative resection. We integrated recurrence of advanced gastric cancer associated miRNA expression profile, targeted genes and gene expression profile and obtain a two-gene classifier to predict recurrence.

Until now, the genes designated in this study to predict the risk of GC recurrence have not been well-characterized. There were no research articles reported on the differential expression in human cancer and no reports on their expression related with microRNAs. HNRPA0 belongs to the A/B subfamily of ubiquitously expressed heterogeneous nuclear ribonucleoproteins (hnRNPs). Gene Ontology (GO) analyses show that HNRPA0 participated in the process of 3'-UTR-mediated mRNA stabilization and regulated the expression of downstream genes.

The protein encoded by PRDM4 is a transcription factor of the PR-domain protein family. Transcription factors of the PR-domain family are known to be involved in cell differentiation and tumorigenesis. An elevated expression level of this gene has been observed in PC12 cells treated with nerve growth factor, $\beta$ polypeptide and it also has been reported to act as a tumor suppressor (34). Similar results were confirmed by GO analyses: PRDM4 acts as a tumor suppressor in negatively regulating cell growth, cell cycle and cell proliferation. Although the functional role of PRDM4 in gastric cancer is unknown, our findings are encouraging.

In summary, we identified a two-gene classifier which can predict recurrence in patients with advanced gastric cancer after curative resection. By combining this classifier with conventional clinicopathological factors, we aim to predict the patient outcome more accurately. The identification of high-risk patients would lead to consideration of additional therapeutic intervention and may be informative for selection of a better follow-up program.

\section{Acknowledgments}

We thank Professor Jiangeng Li (Academy of Electronic Information and Control Engineering, Beijing University of Technology, China) for providing assistance in processing the microarray data with machine learning algorithms. We also thank the Department of Pathology of the Wuhan General Hospital of Guangzhou Command for providing the gastric cancer samples in this study.

\section{References}

1. Yang L: Incidence and mortality of gastric cancer in China. World J Gastroenterol 12: 17-20, 2006.

2. Macdonald JS, Smalley SR, Benedetti J, et al: Chemoradiotherapy after surgery compared with surgery alone for adenocarcinoma of the stomach or gastroesophageal junction. N Engl J Med 345: 725-730, 2001.

3. Lehnert T, Rudek B, Buhl K, et al: Surgical therapy for locoregional recurrence and distant metastasis of gastric cancer. Eur J Surg Oncol 28: 455-461, 2002.

4. Zhang X, Yan Z, Zhang J, et al: Combination of hsa-miR-375 and hsa-miR-142-5p as a predictor for recurrence risk in gastric cancer patients following surgical resection. Ann Oncol 22: 2257-2266, 2011.

5. Roukos DH and Kappas AM: Limitations in controlling risk for recurrence after curative surgery for advanced gastric cancer are now well-explained by molecular-based mechanisms. Ann Surg Oncol 8: 620-621, 2001.

6. Fondevila C, Metges JP, Fuster J, et al: p53 and VEGF expression are independent predictors of tumour recurrence and survival following curative resection of gastric cancer. Br J Cancer 90: 206-215, 2004.

7. Marrelli D, De Stefano A, de Manzoni G, et al: Prediction of recurrence after radical surgery for gastric cancer a scoring system obtained from a prospective multicenter study. Ann Surg 241: 247-255, 2005.

8. Inoue H, Matsuyama A, Mimori K, et al: Prognostic score of gastric cancer determined by cDNA microarray. Clin Cancer Res 8: 3475-3479, 2002.

9. Motoori M, Takemasa I and Yano M: Prediction of recurrence in advanced gastric cancer patients after curative resection by gene expression profiling. Int J Cancer 114: 963-968, 2005.

10. Sim SH, Kim YJ, Oh DY, et al: The role of PET/CT in detection of gastric cancer recurrence. BMC Cancer 9: 73, 2009.

11. Patterson TA, Lobenhofer EK, Fulmer-Smentek SB, et al: Performance comparison of one-color and two-color platforms within the MicroArray Quality Control (MAQC) project. Nat Biotechnol 24: 1140-1150, 2006. 
12. Tusher VG, Tibshirani R and Chu G: Significance analysis of microarrays applied to transcriptional responses to ionizing radiation. Proc Natl Acad Sci USA 98: 5116-5121, 2001.

13. Tibshirani RJ, Hastie TJ, Narasimhan B, et al: Diagnosis of multiple cancer types by shrunken centroids of gene expression. Proc Natl Acad Sci USA 99: 6567-6572, 2002.

14. Guyon I, Weston J, Barnhill S, et al: Gene selection for cancer classification using support vector machines. Machine Learning 46: 389-422, 2002.

15. Furey TS, Cristianini N, Duffy N, et al: Support vector machines classification and validation of cancer tissue samples using microarray expression data. Bioinformatics 16: 906-914, 2000.

16. Chen $\mathrm{X}$ and Ishwaran $\mathrm{H}$ : Random forests for genomic data analysis. Genomics 99: 323-329, 2012.

17. Li S, Harner EJ and Adjeroh DA: Random KNN feature selection - a fast and stable alternative to Random Forests. BMC Bioinformatics 12: 450, 2011.

18. Yan Z, Li J, Xiong Y, et al: Identification of candidate colon cancer biomarkers by applying a random forest approach on microarray data. Oncol Rep 28: 1036-1042, 2012.

19. Kenneth JL and Thomas DS: Analysis of relative gene expression data using real-time quantitative PCR and the $2-\Delta \Delta \mathrm{Ct}$ method. Methods 25: 402-408, 2001

20. Tang F, Zhang R, He Y, et al: MicroRNA-125b induces metastasis by targeting STARD13 in MCF-7 and MDA-MB-231 breast cancer cells. PLoS One 7: e35435, 2012.

21. Gregersen LH, Jacobsen A, Frankel LB, et al: microRNA-143 down-regulates Hexokinase 2 in colon cancer cells. BMC Cancer 12: 232, 2012.

22. Dey N, Das F, Ghosh-Choudhury N, et al: microRNA-21 governs TORC1 activation in renal cancer cell proliferation and invasion. PLoS One 7: e37366, 2012.

23. Kumar B, Yadav A, Lang J, et al: Dysregulation of microRNA34a expression in head and neck squamous cell carcinoma promotes tumor growth and tumor angiogenesis. PLoS One 7: e37601, 2012.
24. Poy MN, Eliasson L, Krutzfeldt J, et al: A pancreatic islet-specific microRNA regulates insulin secretion. Nature 432: 226-230, 2004.

25. Chen CZ, Li L, Lodish HF, et al: MicroRNAs modulate hematopoietic lineage differentiation. Science 303: 83-86, 2004.

26. Liang RQ, Li W, Li Y, et al: An oligonucleotide microarray for microRNA expression analysis based on labeling RNA with quantum dot and nanogold probe. Nucl Acids Res 33: e17, 2005.

27. Ralfkiaer U, Hagedorn PH, Bangsgaard N, et al: Diagnostic microRNA profiling in cutaneous T-cell lymphoma (CTCL). Blood 118: 5891-5900, 2011.

28. Yan Z, Xiong Y, Xu W, et al: Identification of hsa-miR-335 as a prognostic signature in gastric cancer. PLoS One 7: e40037, 2012.

29. Augello C, Vaira V, Caruso L, et al: MicroRNA profiling of hepatocarcinogenesis identifies C19MC cluster as a novel prognostic biomarker in hepatocellular carcinoma. Liver Int 32: 772-782, 2012.

30. Ferracin M, Pedriali M, Veronese A, et al: MicroRNA profiling for the identification of cancers with unknown primary tissue-oforigin. J Pathol 225: 43-53, 2011.

31. Lu Y, Govindan R, Wang L, et al: MicroRNA profiling and prediction of recurrence/relapse-free survival in stage I lung cancer. Carcinogenesis 33: 1046-1054, 2012.

32. Liu R, Chen X, Du Y, et al: Serum microRNA expression profile as a biomarker in the diagnosis and prognosis of pancreatic cancer. Clin Chem 58: 610-618, 2012.

33. White NM, Khella HW, Grigull J, et al: miRNA profiling in metastatic renal cell carcinoma reveals a tumour-suppressor effect for miR-215. Br J Cancer 105: 1741-1749, 2011.

34. Yang XH and Huang S: PFM1 (PRDM4), a new member of the PR-domain family, maps to a tumor suppressor locus on human chromosome 12q23-q24.1. Genomics 61: 319-325, 1999. 